\title{
Dynamics of Quarks in a 2D Flux Tube
}

\author{
Andrew V. Koshelkin ${ }^{1, a}$ and Cheuk-Yin Wong ${ }^{2, b}$ \\ ${ }^{1}$ Moscow Institute for Physics and Engineering, Kashirskoye shosse., 31, 115409 Moscow, Russia \\ ${ }^{2}$ Physics Division, Oak Ridge National Laboratory, Oak Ridge, TN 37830, USA
}

\begin{abstract}
On the basis of a compactification of the (3+1) into (1+1) dimensional space-time [1] , the quark states inside the 2D flux tube are studied for the case of a linear transverse confining potential. The derived states are classified by both the projections of the orbital momentum and the spin along the tube direction. The spectrum of the fermion states is evaluated. It is found that the energy eigenvalues of the quarks appear to be approximately related to the square root of the eigenvalues of the two-dimensional harmonic oscillator.
\end{abstract}

\section{Introduction}

The three-dimensional QCD flux tube between a quark and an antiquark and its idealization as a one-dimensional string are useful concepts (for a review see [2]). The flux tube enters into the flux-tube model of hadrons [3], and the QCD string provides the arena for the fragmentation model of particle production $[4,5]$. The idealization of the flux-tube as a string has the limitation that it pertains mainly to the dynamics along the longitudinal direction and cannot describe the dynamics in the transverse directions. To describe the transverse dynamics, it is necessary to examine the structure and the states of particles in the flux tube. The knowledge of these states furnishes useful information for the investigation of the transverse momentum distribution of the produced particles, for which a wealth of information have been collected recently [6-12].

In the flux tube model, the $q$ and $\bar{q}$ partons in the flux tube are assumed to be confined and are subject to transverse confinement. We can describe transverse confinement in terms of a confining scalar interaction $S\left(\mathbf{r}_{\perp}\right)$ in transverse coordinates $\mathbf{r}_{\perp}$, with the quark mass function described by $m\left(\mathbf{r}_{\perp}\right)=m_{0}+S\left(\mathbf{r}_{\perp}\right)$ where $m_{0}$ is the quark rest mass.

Previously, we show from action integral that under the assumption of transverse confinement and longitudinal dominance, $\mathrm{QCD}_{4}$ in $(3+1)$ dimensional space-time can be approximately compactified into $\mathrm{QCD}_{2}$ in $(1+1)$ dimensional space-time [1]. In such a process, we find the equations of motion for quarks in the flux tube, and the relation between the coupling constant $g_{2 \mathrm{D}}$ in $\mathrm{QCD}_{2}$ and the coupling constant $g_{4 \mathrm{D}}=g$ in $\mathrm{QCD}_{4}$. We show how the quark and the gluon in $\mathrm{QCD}_{2}$ acquire masses arising their being confined within the tube, and how all these quantities depend on the transverse wave functions of the quarks in the tube. The variation of the gluon fields leads to an

\footnotetext{
a e-mail: A_Kosh@internets.ru

be-mail: wongc@ornl.gov
}

additional mass that depends on the coupling constant $g_{2 \mathrm{D}}$. The compactification facilitates the investigation of some problems in $\mathrm{QCD}_{4}$ in the simpler dynamics of $\mathrm{QCD}_{2}$ and brings us to a better understanding of the different degrees of freedom of the produced particles.

\section{Equations of Motion for Transverse Dynamics of Quarks}

We write the fermion field $\Psi(x)$ in terms of the following spinor with transverse functions $G_{ \pm}\left(\mathbf{r}_{\perp}\right)$ and $x^{0}-x^{3}$ functions $f_{ \pm}\left(x^{0}, x^{3}\right)[1,13]$,

$$
\Psi(x)=\frac{1}{\sqrt{2}}\left(\begin{array}{c}
G_{+}\left(\mathbf{r}_{\perp}\right)\left(f_{+}\left(x^{0} ; x^{3}\right)+f_{-}\left(x^{0} ; x^{3}\right)\right) \\
-G_{-}\left(\mathbf{r}_{\perp}\right)\left(f_{+}\left(x^{0} ; x^{3}\right)-f_{-}\left(x^{0} ; x^{3}\right)\right) \\
G_{+}\left(\mathbf{r}_{\perp}\right)\left(f_{+}\left(x^{0} ; x^{3}\right)-f_{-}\left(x^{0} ; x^{3}\right)\right) \\
G_{-}\left(\mathbf{r}_{\perp}\right)\left(f_{+}\left(x^{0} ; x^{3}\right)+f_{-}\left(x^{0} ; x^{3}\right)\right)
\end{array}\right),
$$

where we use the notations of [1], and $\mathbf{r}_{\perp}=\left(x^{1}, x^{2}\right)$ is a vector in the plane perpendicular to the $x^{3}$ axis. To obtain the equations of motion for the functions $G_{+}\left(\mathbf{r}_{\perp}\right)$ and $G_{-}\left(\mathbf{r}_{\perp}\right)$, we vary the action integral $\mathcal{A}(4 D)$ in Eq. (11) of [1], under the constraint of the normalization condition. To do this we construct a new functional $\mathcal{A}^{\prime}$ :

$$
\begin{aligned}
\mathcal{A}^{\prime}(4 D)= & \mathcal{A}(4 D)+\frac{\lambda^{2}}{2} \int d x^{1} d x^{2}\left(\left|G_{+}\left(\mathbf{r}_{\perp}\right)\right|^{2}+\left|G_{-}\left(\mathbf{r}_{\perp}\right)\right|^{2}\right) \\
& \times \int d x^{0} d x^{3}\left(\bar{\psi}(2 D, X) m_{q T} \psi(2 D, X)\right)
\end{aligned}
$$

where $\lambda^{2}$ is the Lagrange multiplier; $\psi(2 D, X)$ and $m_{q T}$ are the $2 \mathrm{D}$ spinor and the fermion effective mass in the $(1+1)$ space-time [1]. The last term in Eq. (2) takes into account the unitarity of a fermion field in the 4D spacetime. Varying $\mathcal{A}^{\prime}$ with respect to the functions $G_{+}\left(\mathbf{r}_{\perp}\right)$ and $G_{-}\left(\mathbf{r}_{\perp}\right)$, we derive

$$
\begin{aligned}
& \left(p_{1}+i p_{2}\right) G_{+}\left(\mathbf{r}_{\perp}\right)=\left(m\left(\mathbf{r}_{\perp}\right)+\lambda^{2}\right) G_{-}\left(\mathbf{r}_{\perp}\right), \\
& \left(p_{1}-i p_{2}\right) G_{-}\left(\mathbf{r}_{\perp}\right)=\left(\lambda^{2}-m\left(\mathbf{r}_{\perp}\right)\right) G_{+}\left(\mathbf{r}_{\perp}\right) .
\end{aligned}
$$


Combining the above two equations, we obtain

$\left(p_{1}^{2}+p_{2}^{2}-\lambda^{2}+m^{2}\left(\mathbf{r}_{\perp}\right)\right) G_{+}\left(\mathbf{r}_{\perp}\right)=G_{-}\left(\mathbf{r}_{\perp}\right)\left(p_{1}-i p_{2}\right) m\left(\mathbf{r}_{\perp}\right)$,

$\left(p_{1}^{2}+p_{2}^{2}-\lambda^{2}+m^{2}\left(\mathbf{r}_{\perp}\right)\right) G_{-}\left(\mathbf{r}_{\perp}\right)=-G_{+}\left(\mathbf{r}_{\perp}\right)\left(p_{1}+i p_{2}\right) m\left(\mathbf{r}_{\perp}\right)$.

We have also

$$
p_{1} \mp i p_{2}=e^{\mp i \varphi}\left[i \frac{d}{d r} \pm \frac{1}{r} \frac{d}{d \varphi}\right] .
$$

The general equations coupling $G_{+}\left(\mathbf{r}_{\perp}\right)$ and $G_{-}\left(\mathbf{r}_{\perp}\right)$ for a scalar mass function $m\left(\mathbf{r}_{\perp}\right)$ in transverse confinement are

$\left(\mathbf{p}_{\perp}^{2}-\lambda^{2}+m^{2}\left(\mathbf{r}_{\perp}\right)\right) G_{+}\left(\mathbf{r}_{\perp}\right)=G_{-}\left(\mathbf{r}_{\perp}\right) e^{-i \varphi}\left[i \frac{d}{d r}+\frac{1}{r} \frac{d}{d \varphi}\right] m\left(\mathbf{r}_{\perp}\right)$,

$\left(\mathbf{p}_{\perp}^{2}-\lambda^{2}+m^{2}\left(\mathbf{r}_{\perp}\right)\right) G_{-}\left(\mathbf{r}_{\perp}\right)=-G_{+}\left(\mathbf{r}_{\perp}\right) e^{+i \varphi}\left[i \frac{d}{d r}-\frac{1}{r} \frac{d}{d \varphi}\right] m\left(\mathbf{r}_{\perp}\right)$.

The above equations show that $\lambda^{2}$ plays the role of the fermion eigenenergy.

\section{Axisymmetric Potential}

In this manuscript, we consider an axisymmetric linearly confining potential

$$
m\left(\mathbf{r}_{\perp}\right)=\kappa\left|\mathbf{r}_{\perp}\right|,
$$

where the parameter $\kappa$ can be interpreted as the string tension in the radial direction of the flux tube. Then, the set of coupled equations become

$$
\begin{aligned}
& \left(\mathbf{p}_{\perp}^{2}+m^{2}\left(r_{\perp}\right)\right) G_{+}\left(\mathbf{r}_{\perp}\right)-i \kappa e^{-i \varphi} G_{-}\left(\mathbf{r}_{\perp}\right)=\lambda^{2} G_{+}\left(\mathbf{r}_{\perp}\right), \\
& \left(\mathbf{p}_{\perp}^{2}+m^{2}\left(r_{\perp}\right)\right) G_{-}\left(\mathbf{r}_{\perp}\right)+i \kappa e^{+i \varphi} G_{+}\left(\mathbf{r}_{\perp}\right)=\lambda^{2} G_{-}\left(\mathbf{r}_{\perp}\right) .
\end{aligned}
$$

In matrix form they are

$$
\left(\begin{array}{cc}
H_{0} & -i \kappa e^{-i \varphi} \\
+i \kappa e^{+i \varphi} & H_{0}
\end{array}\right)\left(\begin{array}{l}
G_{+}\left(\mathbf{r}_{\perp}\right) \\
G_{-}\left(\mathbf{r}_{\perp}\right)
\end{array}\right)=\lambda^{2}\left(\begin{array}{l}
G_{+}\left(\mathbf{r}_{\perp}\right) \\
G_{-}\left(\mathbf{r}_{\perp}\right)
\end{array}\right)
$$

or

$$
H G=\lambda^{2} G
$$

where

$$
H=\left(\begin{array}{cc}
H_{0} & -i \kappa e^{-i \varphi} \\
+i \kappa e^{+i \varphi} & H_{0}
\end{array}\right), \quad G=\left(\begin{array}{l}
G_{+}\left(\mathbf{r}_{\perp}\right) \\
G_{-}\left(\mathbf{r}_{\perp}\right)
\end{array}\right) .
$$

The Hamiltonian $H_{0}$ is given by

$$
H_{0}=\left(-\frac{1}{r_{\perp}} \frac{\partial}{\partial r_{\perp}} r_{\perp} \frac{\partial}{\partial r_{\perp}}-\frac{1}{r_{\perp}^{2}} \frac{\partial^{2}}{\partial \varphi^{2}}+\kappa^{2} r_{\perp}^{2}\right)
$$

Each fermion eigenstate is characterized by the $z$ component of the total angular momentum $\Omega$. To separation out the $\varphi$ and $r_{\perp}$ dependencies, we introduce the radial wave function $\mathcal{R}_{\Omega \sigma}\left(r_{\perp}\right)$ to write wave function $G_{ \pm}$as

$$
G_{\Omega \sigma}\left(r_{\perp}, \varphi\right)=i^{(1-\sigma) / 2} \frac{e^{i(\Omega-\sigma / 2) \varphi}}{\sqrt{2 \pi}} \mathcal{R}_{\Omega \sigma}\left(r_{\perp}\right),
$$

where $\sigma= \pm 1$. Then, we obtain from Eq.(9)

$$
\left(\begin{array}{cc}
H_{0}(\Omega-1 / 2) & \kappa \\
\kappa & H_{0}(\Omega+1 / 2)
\end{array}\right)\left(\begin{array}{l}
\mathcal{R}_{\Omega+}\left(\mathbf{r}_{\perp}\right) \\
\mathcal{R}_{\Omega-}\left(r_{\perp}\right)
\end{array}\right)=\lambda^{2}\left(\begin{array}{l}
\mathcal{R}_{\Omega+}\left(\mathbf{r}_{\perp}\right) \\
\mathcal{R}_{\Omega-}\left(\mathbf{r}_{\perp}\right)
\end{array}\right) .
$$

Here, the hamiltonian $H_{0}(\Omega \mp 1 / 2)$ is

$H_{0}(\Omega \mp 1 / 2)=\left(-\frac{1}{r_{\perp}} \frac{\partial}{\partial r_{\perp}} r_{\perp} \frac{\partial}{\partial r_{\perp}}+\frac{(\Omega \mp 1 / 2)^{2}}{r_{\perp}^{2}}+\kappa^{2} r_{\perp}^{2}\right)$.

\section{Eigenvalue equation}

For fermions, $\Omega$ are positive and negative half-integers. Eqs. (13)-(15) suggests the usefulness of expanding the radial wave function $\mathcal{R}_{\Omega \sigma}$ in terms of a complete set of Harmonic oscillator radial basis $R_{n \Omega \sigma}$. Accordingly, we expand $|G(\Omega)\rangle$ in terms of a complete set of Harmonic oscillator states $|n \Omega \sigma\rangle$ with specific phase factors as

$$
|G(\Omega)\rangle=\sum_{n \sigma} C_{n \Omega \sigma}|n \Omega \sigma\rangle=\sum_{n \sigma} C_{n \Omega \sigma} \psi_{n \Omega \sigma}(\xi, \varphi) \chi_{\sigma}
$$

where

$$
\begin{aligned}
& \chi_{+}=\left(\begin{array}{l}
1 \\
0
\end{array}\right), \quad \text { and } \chi_{-}=\left(\begin{array}{l}
0 \\
1
\end{array}\right), \\
& \psi_{n \Omega \sigma}(\xi, \varphi)=i^{(1-\sigma) / 2} \frac{e^{i(\Omega-\sigma / 2) \varphi}}{\sqrt{2 \pi}} R_{n \Omega \sigma}(\xi) .
\end{aligned}
$$

Here, the radial basis state wave functions $R_{n \Omega \sigma}(\xi)$ are

$$
R_{n \Omega \sigma}(\xi)=N_{n \Omega \sigma} e^{-\xi / 2} \xi^{|\Omega-\sigma / 2| / 2} L_{n}^{|\Omega-\sigma / 2|}(\xi),
$$

where $\xi=\kappa r_{\perp}^{2}$, and the normalization constant is

$$
N_{n \Omega \sigma}=\sqrt{\frac{2 \kappa n !}{\Gamma(|\Omega-\sigma / 2|+n+1)}} .
$$

The basis states $|n \Omega \sigma\rangle=\psi_{n \Omega \sigma} \chi_{\sigma}$ are orthonormal,

$$
\begin{aligned}
\left\langle n^{\prime} \Omega^{\prime} \sigma^{\prime} \mid n \Omega \sigma\right\rangle & =\left\langle\psi_{n^{\prime} \Omega^{\prime} \sigma^{\prime}}(\xi, \varphi) \chi_{\sigma^{\prime}} \mid \psi_{n \Omega \sigma}(\xi, \varphi) \chi_{\sigma}\right\rangle \\
& =\delta_{n^{\prime} n} \delta_{\Omega^{\prime} \Omega} \delta_{\sigma^{\prime} \sigma} .
\end{aligned}
$$

Substitute the expansion (16) into Eq. (10), we can separate out the $\varphi$ degrees of freedom and get

$$
\begin{gathered}
\sum_{n=0}^{\infty}\left(\begin{array}{cr}
H_{0}(\Omega-1 / 2) & \kappa \\
\kappa & H_{0}(\Omega+1 / 2)
\end{array}\right)\left[C_{n \Omega+} R_{n \Omega+}\left(\begin{array}{l}
1 \\
0
\end{array}\right)+C_{n \Omega-} R_{n \Omega-}\left(\begin{array}{l}
0 \\
1
\end{array}\right)\right] \\
=\lambda^{2} \sum_{n=0}^{\infty}\left[C_{n \Omega+} R_{n \Omega+}\left(\begin{array}{l}
1 \\
0
\end{array}\right)+C_{n \Omega-} R_{n \Omega-}\left(\begin{array}{l}
0 \\
1
\end{array}\right)\right],
\end{gathered}
$$

where the subscripts " $( \pm)$ " refer to the $\sigma= \pm 1$ quantum numbers and $H_{0}(\Omega \mp 1 / 2)$ is given by Eq.(15). So, the eigenvalue equation becomes the following set of coupled equations:

$$
\begin{aligned}
& \sum_{n=0}^{\infty}\left\{H_{0}(\Omega-1 / 2) C_{n \Omega+} R_{n \Omega+}+\kappa C_{n \Omega-} R_{n \Omega-}\right\}=\lambda^{2} \sum_{n=0}^{\infty} C_{n \Omega+} R_{n \Omega+}, \\
& \sum_{n=0}^{\infty}\left\{H_{0}(\Omega+1 / 2) C_{n \Omega-} R_{n \Omega-}+\kappa C_{n \Omega+} R_{n \Omega+}\right\}=\lambda^{2} \sum_{n=0}^{\infty} C_{n \Omega-} R_{n \Omega-} .
\end{aligned}
$$

We note that we have chosen $R_{n \Omega \sigma}$ to be the radial eignestates of $H_{0}(\Omega \mp \sigma / 2)$. It satisfies the eigenvalue equation

$$
\begin{gathered}
H_{0}(\Omega \mp \sigma / 2) R_{n \Omega \sigma}=\kappa[4 n+2|\Omega \mp \sigma / 2|+2] R_{n \Omega \sigma} \\
\equiv \kappa \epsilon^{2}(n \Omega \sigma) R_{n \Omega \sigma} .
\end{gathered}
$$

where we have introduced dimensionless $\epsilon^{2}$ as

$$
\epsilon^{2}(n \Omega \sigma)=[4 n+2|\Omega \mp \sigma / 2|+2] .
$$


Upon taking the scalar product with $R_{n \Omega+}$ for the first equation in Eq. (21) and with $R_{\Omega n-}$ for the second equation, we get

$C_{n \Omega+}\left\{\kappa \epsilon^{2}(n \Omega+)-\lambda^{2}\right\}+\kappa \sum_{m=0}^{\infty} C_{m \Omega-}\langle n \Omega+, m \Omega-\rangle=0$,

$C_{n \Omega-}\left\{\kappa \epsilon^{2}(n \Omega-)-\lambda^{2}\right\}+\kappa \sum_{m=0}^{\infty} C_{m \Omega+}\langle n \Omega-, m \Omega+\rangle=0$,

where the scalar product $\left\langle n \Omega \sigma^{\prime}, \Omega m \sigma\right\rangle$ involves only the radial wave functions,

$$
\left\langle n \Omega \sigma^{\prime}, m \Omega \sigma\right\rangle=\int \pi d \xi R_{n \Omega \sigma^{\prime}}^{*}(\xi) R_{m \Omega \sigma}(\xi) .
$$

The set of coupled equations in Eq. (24) for $C_{n \Omega \sigma}$ can be written as a matrix eigenvalue equation in the form

$$
\mathcal{H} G(\Omega)=\frac{\lambda^{2}}{\kappa} G(\Omega),
$$

where $G(\Omega) \equiv|G(\Omega)\rangle$ is a column vector of coefficients $C_{\Omega n \sigma}$ with known basis states $|n \Omega \sigma\rangle$,

$$
G(\Omega)=\left(\begin{array}{c}
C_{0 \Omega+} \\
C_{0 \Omega-} \\
C_{1 \Omega+} \\
C_{1 \Omega-} \\
\ldots
\end{array}\right),
$$

and $\mathcal{H}$ is the matrix

$$
\left(\begin{array}{ccccc}
\epsilon^{2}(0 \Omega+) & \langle 0 \Omega+, 0 \Omega-\rangle & 0 & \langle 0 \Omega+, 1 \Omega-\rangle & \ldots \\
\langle 0 \Omega-, \Omega 0+\rangle & \epsilon^{2}(0 \Omega-) & \langle 0 \Omega-, 1 \Omega+\rangle & 0 & \ldots \\
0 & \langle 1 \Omega+, 0 \Omega-\rangle & \epsilon^{2}(1 \Omega+) & \langle 1 \Omega+, 1 \Omega-\rangle & \ldots \\
\langle 1 \Omega-, 0 \Omega+\rangle & 0 & \langle 1 \Omega-, 1 \Omega+\rangle & \epsilon^{2}(1 \Omega-) & \ldots \\
\ldots & \ldots & \ldots & \ldots & \ldots
\end{array}\right) .
$$

The matrix equation (26) can be diagonalized numerically. After an eigenvalue $\lambda^{2} / \kappa$ is obtained, the corresponding eigenstate $|G(\Omega)\rangle$ can be normalized by choosing a normalization factor for the $C_{n \Omega \sigma}$ coefficients such that

$$
\langle G(\Omega) \mid G(\Omega)\rangle=\sum_{n \sigma}\left|C_{n \Omega \sigma}\right|^{2}=1 .
$$

We note that the set of equations in (24) is invariant when we interchange $\Omega \leftrightarrow(-\Omega)$ and $\sigma \leftrightarrow(-\sigma)$. Thus, the $\pm \Omega$ are doubly degenerate with

$$
\lambda^{2}(\Omega)=\lambda^{2}(-\Omega),
$$

and the wave functions are related to each other,

$$
C_{n-\Omega \sigma}=C_{n} \Omega-\sigma .
$$

Therefore, without losses of generality, we can focus our attention on positive $\Omega=1 / 2,3 / 2,5 / 2, \ldots$

As an illustration, it is illuminating to obtain the approximate eigenvalues and eigenfunctions when we consider the following simplifying approximation

$$
\langle n \Omega+, m \Omega-\rangle=\delta_{n m},
$$

then the original full Hamiltonian will be truncated and we obtain the approximate eigenvalue equation,

$$
\left(\begin{array}{cc}
\epsilon^{2}(n \Omega+) & \langle n \Omega+, n \Omega-\rangle \\
\langle n \Omega-, n \Omega+\rangle & \epsilon^{2}(n \Omega-)
\end{array}\right)\left(\begin{array}{l}
C_{n \Omega+} \\
C_{n \Omega-}
\end{array}\right)=\frac{\lambda^{2}}{\kappa}\left(\begin{array}{l}
C_{n \Omega+} \\
C_{n \Omega-}
\end{array}\right) .
$$

This eigenvalue equation is of the form

$$
\begin{aligned}
& \left(\begin{array}{cc}
\bar{E}+\Delta & V \\
V & \bar{E}-\Delta
\end{array}\right)\left(\begin{array}{l}
C_{n \Omega+} \\
C_{n \Omega-}
\end{array}\right)=\lambda^{2}\left(\begin{array}{l}
C_{n \Omega+} \\
C_{n \Omega-}
\end{array}\right) \\
& \bar{E}=\frac{\epsilon^{2}(n \Omega+)+\epsilon^{2}(n \Omega-)}{2} \\
& \Delta=\frac{\epsilon^{2}(n \Omega+)-\epsilon^{2}(n \Omega-)}{2},
\end{aligned}
$$

with eigenvalues

$$
\lambda^{2}=\bar{E} \pm \sqrt{V^{2}+\Delta^{2}}
$$

and (unnormalized) eigenvectors

$$
C_{1}=\left(\begin{array}{c}
\Delta+\sqrt{V^{2}+\Delta^{2}} \\
-V
\end{array}\right), C_{2}=-\left(\begin{array}{c}
V \\
\Delta+\sqrt{V^{2}+\Delta^{2}}
\end{array}\right) .
$$

When $V$ is zero, the state $C_{1}$ will be a purely $|n \Omega(\sigma=1 / 2)\rangle$ state and the state $C_{2}$ will be a purely $\mid n \Omega(\sigma=-1 / 2\rangle$ state.

\section{Numerical Solutions}

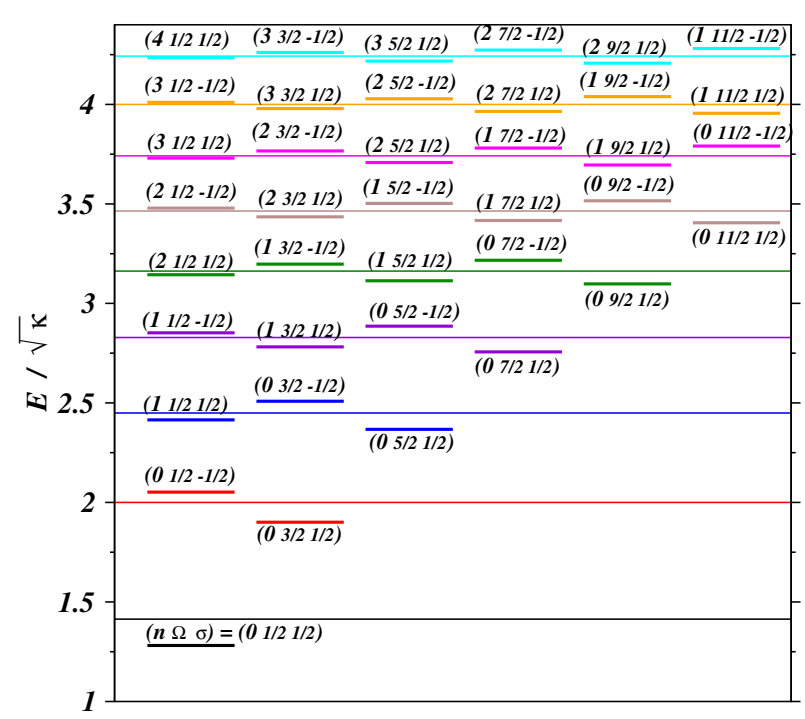

Figure 1. (Color online) The spectrum of transverse fermion states of different $\Omega$ in a flux tube. The label on each energy level represents the quantum numbers $(n, \Omega, \sigma)$ of its dominant component. The long horizontal lines are the energy levels given by Eq. (23), namely, $E / \sqrt{\kappa}=\sqrt{4 n+2|\Omega-\sigma / 2|+2}$.

The Hamiltonian for the eigenvalue equation (26) can be constructed and the eigenvalues and eigenvectors obtained by numerical Gauss-Laguerre integration and by matrix diagonalization. The analysis of the Hamiltonian in the matrix representation shows that the matrix element $H(i j) \ll H(i i)$ for $i \neq j$ and furthermore, $H(i j)$ decreases as the difference between $i$ and $j$ increases. The eignevalues $\lambda^{2} / \kappa$ is therefore dominated by the diagonal matrix elements.

The eigenstate energy $E$ is the square root of $\lambda^{2}$ and we have

$$
E / \sqrt{\kappa}= \pm \sqrt{\lambda^{2} / \kappa}
$$


possessing both the positive and negative energy states. The positions of the lowest positive energy states are presented in Fig.1. Each state is doubly degenerate and is labelled by the quantum numbers $(n, \Omega, \sigma)$ of its dominant component. An eigenstate wave function is therefore given approximately by

$$
|n \Omega \sigma\rangle=\psi(n \Omega \sigma) \chi_{\sigma}=i^{(1-\sigma) / 2} \frac{e^{i(\Omega-\sigma / 2) \varphi}}{\sqrt{2 \pi}} R_{n \Omega \sigma}\left(r_{\perp}\right) \chi_{\sigma},
$$

where $R_{n \Omega \sigma}$ is given by Eq. (19). It is reasonable to approximate the corresponding energy eigenvalue as

$$
E(n \Omega \sigma) \sim \pm \sqrt{4 n+2|\Omega-\sigma / 2|+2} \sqrt{\kappa} .
$$

In Fig. 1, the long horizontal lines are the positive energy levels given by the above approximate eigenvalues, which are close to the eigenvalues obtained by numerical diagonalization. Thus, the spectrum of $E$ is related approximately to the square root of the eigenvalues of the twodimensional harmonic oscillator, where the radial quantum number $n$ have become approximately a "good" quantum number.

\section{Effective Masses of Quarks and Gluons and 2D coupling constant}

It was shown in [1] that the quark effective mass $m_{q T}$ in the 2D flux tube considerations coincides with the eigenvalue $\lambda$. For the approximate eigenstate (37) with the dominant component of $|n \Omega \sigma\rangle$, we therefore have

$$
m_{q T}(n \Omega \sigma) \sim|E(n \Omega \sigma)| \sim \sqrt{4 n+2|\Omega-\sigma / 2|+2} \sqrt{\kappa} .
$$

As for the effective gluon mass $m_{g T}$ generated by $Q C D_{4} \rightarrow Q C D_{2}$ compactification, it is proportional to the kinetic energy term of the Hamiltonian $H_{0}$ given by Eq.(10). Since the mean kinetic energy of an oscillator is one half of its total energy, we have

$$
m_{g T}(n \Omega \sigma) \sim \frac{1}{2} \sqrt{4 n+2|\Omega-\sigma / 2|+2} \sqrt{\kappa} .
$$

In the flux tube consideration the $g_{2 D}$ coupling constant is [1]:

$$
g_{2 D}(n \Omega \sigma)=\sum_{\sigma} \int d x^{1} d x^{2} g_{4 D}\left[\left|G_{\sigma}\left(\mathbf{r}_{\perp}\right)\right|^{2}\right]^{3 / 2}
$$

Substituting the wave functions given by Eq. (37) into the last formula, we obtain for $n=0$ :

$$
g_{2 D} \sim \frac{2 g \kappa^{1 / 2}}{3 \sqrt{\pi}} \sum_{\sigma}\left(\frac{2^{|\Omega-\sigma / 2|} \Gamma^{2 / 3}(3 / 2|\Omega-\sigma / 2|+1)}{3^{|\Omega-\sigma / 2|} \Gamma(|\Omega-\sigma / 2|+1)}\right)^{3 / 2}
$$

The total effective gluon mass $M_{t T}$ which is induced by the $Q C D_{4} \rightarrow Q C D_{2}$ compactification and interaction between 2D fermions and gluons is given by the formula [1]:

$$
M_{t T}=\sqrt{m_{g T}^{2}+\frac{g_{2 D}^{2} N_{f}}{2 \pi}},
$$

where $N_{f}$ is the number of flavors.

\section{Conclusion}

On the basis of the $Q C D_{4} \rightarrow Q C D_{2}$ compactification [1], we study the quark states in an axial symmetric radiallyconfining potential. The eigenvalues and eigenvectors of these state are evaluated and the spectrum of the eigenstates is obtained by diagonalizing the exact Hamiltonian. The results of the calculations show that the energy spectrum is related approximately to the square root of the eigenvalues of the two-dimensional harmonic oscillator.

The obtained fermion eigenstates allow us to calculate the effective masses of quarks and gluons in the $2 \mathrm{D}$ flux tube, as well as the coupling constant in the compactified $\mathrm{QCD}_{2}$ [1]. Following the string fragmentation concept [2, 4], they will enable us to derive the $p_{T}$ distribution of the observable hadrons arising from the flux tubes formed in the collisions of high energy proton and nuclear collisions.

\section{Acknowledgement}

This research was supported in part by the Division of Nuclear Physics, U.S. Department of Energy, under Contract No. DE-AC05-00OR22725.

\section{References}

[1] A. V. Koshelkin, C. Y. Wong, Phys. Rev. D86 (2012) 125026.

[2] C. Y. Wong, Introduction to High-Energy Heavy-Ion Collisions, World Scientific Publisher, 1994.

[3] N. Isgur and J. Paton, Phys. Rev. D31, 2910 (1985).

[4] A. Casher, J. Kogut, and L. Susskind, Phys. Rev. D10, 732 (1974).

[5] B. Andersson, G. Gustafson, and T. Sjöstrand, Zeit. für Phys. C20, 317 (1983); B. Andersson, G. Gustafson, G. Ingelman, and T. Sjöstrand, Phys. Rep. 97, 31 (1983); T. Sjöstrand and M. Bengtsson, Computer Physics Comm. 43, 367 (1987); B. Andersson, G. Gustavson, and B. Nilsson-Alqvist, Nucl. Phys. B281, 289 (1987).

[6] B. I. Abelev et al. (STAR Collaboration), Phys. Rev. C 75 (2007) 064901.

[7] A. Adare et al. (PHENIX Collaboration), Phys. Rev. D 83 (2011) 024909, 052004 and 064903.

[8] G. Aad et al. (ATLAS Collaboration), New J. Phys. 13 (2011) 053033.

[9] V. Khachatryan et al. (CMS Collaboration), JHEP 02 (2010) 041 and Phys. Rev. Lett. 105 (2010) 022002.

[10] V. Khachatryan et al. (CMS Collaboration), JHEP 08 (2011) 086.

[11] K. Aamodt et al. (ALICE Collaboration), Phys. Lett. B 693 (2010) 53; Eur. Phys. J. C 71 (2011) 1594 and 1655.

[12] C. Y. Wong, G. Wilk, Acta Phys. Pol. B 43, 2047 (2012).

[13] C. Y. Wong, R. C. Wang, and C. C. Shih, Phys. Rev. D 44, 257 (1991). 